\title{
Genetic Analysis Indicates Maize Pullulanase- and Isoamylase-type Starch Debranching Enzymes Have Partially Overlapping Functions in Starch Metabolism
}

\author{
(Received September 15, 2002) \\ Jason R. Dinges, ${ }^{1}$ Martha G. James ${ }^{1}$ and Alan M. Myers ${ }^{1, *}$ \\ ${ }^{1}$ Department of Biochemistry, Biophysics, and Molecular Biology, Iowa State University \\ (1210, Molecular Biology Building, Ames, IA 50011, USA)
}

\begin{abstract}
The functions of the two known plant classes of $\alpha(1 \rightarrow 6)$ glucan hydrolase were investigated by genetic analysis in maize. A null mutation in the zpul gene, coding for the pullulanase-type starch debranching enzyme (DBE), was coupled with three different alleles of the sul gene, coding for the isoamylase-type DBE. In two instances the starch biosynthesis abnormality conditioned by the isoamylase-type DBE mutation, namely a decrease in starch content and an increase in phytoglycogen content, was exacerbated by the additional loss of pullulanase-type DBE activity. These data confirm previous findings that pullulanase-type DBE contributes to starch biosynthesis in developing endosperm. An appreciable level of starch was still formed, however, in mutant endosperm bearing null mutations for both classes of DBE, and in this instance the pullulanase-type DBE had no effect on the amount of starch produced. The data suggest that a critical level of isoamylase-type DBE protein or activity may be required in order for pullulanase-type DBE to be able to function productively in starch biosynthesis. Furthermore, the data show clearly that a significant degree of starch biosynthesis, approximately $40 \%$ of normal, occurs in the absence of any detectable DBE activity in the endosperm of maize kernels.
\end{abstract}

Key words: isoamylase, phytoglycogen, pullulanase, starch debranching enzyme, sugary 1

This study investigated the physiological functions of starch debranching enzymes (DBEs), which hydrolyze $\alpha(1 \rightarrow 6)$ glycoside bonds in glucose polymers. Plant $\alpha(1$ $\rightarrow 6$ ) glucan hydrolases can be broadly classified as either "isoamylase-type DBEs" or "pullulanase-type DBEs" (also known as limit dextrinases, or R-enzymes), based upon their in vitro substrate specificity as well as amino acid similarity to previously characterized prokaryotic enzymes. Isoamylase-type DBEs hydrolyze $\alpha(1 \rightarrow 6)$ bonds in glycogen and amylopectin, but are not active towards such linkages in pullulan. Pullulanase-type DBEs, in contrast, readily hydrolyze $\alpha(1 \rightarrow 6)$ bonds in pullulan, but are inactive or only very slightly active towards glycogen or amylopectin. ${ }^{1-3)}$ Genomic sequences reveal three distinct genes that code for isoamylase-type DBEs in rice and Arabidopsis, and three distinct cDNAs coding for such enzymes are known in maize and potato. ${ }^{4-6)} \mathrm{A}$ single gene coding for pullulanase-type DBE has been identified in the two available genomic sequences, and a single pullulanase-type DBE cDNA has been isolated from maize and other plants. ${ }^{4,5,7-10)}$ Phylogenetic analysis shows that isoamylase- and pullulanase-type DBEs have been conserved separately throughout the evolution of plants and bacteria. ${ }^{8)}$ These data support the hypothesis that the two different types of DBE, despite having the same enzymatic activity, have specific physiological functions that cannot be completely substituted by a member of the

${ }^{*}$ Corresponding author (Tel. +1-515 294-6116, Fax. + 1-515 294-0453, E-mail: ammyers@iastate.edu).

Abbreviations: DBE, debranching enzyme; PG, phytoglycogen; WSP, water soluble polysaccharide. other class. The specific function(s) of each DBE class, however, remains to be discerned.

The hydrolytic activity of the DBEs points to an obvious role in starch degradation, but extensive genetic and biochemical analysis in many plant species has shown that mutations in these enzymes affect starch anabolism as well as its breakdown. Mutations in one of the isoamylasetype DBE genes, coded for by the sugaryl (sul) gene of maize $^{11)}$ and rice ${ }^{12)}$ and its orthologs in Arabidopsis, ${ }^{13)}$ barley ${ }^{14)}$ and Chlamydomonas, ${ }^{15)}$ result in decreased starch amounts and accumulation of a highly-branched, water soluble polysaccharide (WSP) termed phytoglycogen (PG). Three models have been proposed to explain the accumulation of PG in DBE mutants and, accordingly, the function of these enzymes in starch synthesis. The glucan trimming model proposes that DBEs are directly involved in the determination of the amylopectin (Ap) fine structure by removing extraneous branches from a nascent Ap chain, thereby facilitating crystallization. ${ }^{16,17)}$ In the absence of such activity, branching may reach a level where crystallization is no longer possible, leading to PG accumulation. A second model suggests that DBEs may be involved in starch synthesis indirectly by clearing soluble glucan, i.e., polymer accumulating separately from the granule, that would otherwise divert enzyme and substrate from the productive synthesis occurring at the granule surface. ${ }^{13,18)}$ A third model recently was proposed that suggests the DBEs are necessary for proper starch granule initiation. ${ }^{14)}$ Uncontrolled granule initiation could lead to multiple, compound granules and phytoglycogen accumulation. 
The pullulanase- and isoamylase-type DBEs may be functionally related. Studies of $s u 1^{-}$mutants of maize and rice revealed that loss of isoamylase-type DBE causes a concomitant reduction in the activity of the pullulanasetype DBE as a secondary, pleiotropic effect. ${ }^{3,8,19)}$ By correlating starch-containing regions of $\mathrm{sul}^{-}$mutant rice endosperm with residual pullulanase-type DBE activity, Nakamura et al. ${ }^{20,21)}$ speculated that the pullulanase-type $\mathrm{DBE}$, not the isoamylase-type $\mathrm{DBE}$, is the primary determinant of the phytoglycogen accumulating phenotype. Pleiotropic effects on pullulanase-type DBE activity, however, do not result from isoamylase mutations in Arabidopsis, barley, or Chlamydomonas. ${ }^{13-15)} \mathrm{Wu}$ et al. have speculated that the reduction of pullulanase-type DBE activity in isoamylase-type DBE mutants is caused by posttranslational modification of the enzyme, perhaps resulting from increased cellular sucrose concentrations. ${ }^{3)}$

The functional relationships between the two types of DBE were addressed recently by use of a transposon insertion mutation in the gene zpul. ${ }^{6)}$ The mutant allele zpul-204 caused complete loss of pullulanase-type DBE activity, and resulted in defects in starch degradation in leaves as well as germinating seeds. The mutation alters the metabolism of maltooligosaccharides (MOS) in the developing kernel, but does not lead to production of detectable quantities of PG. In itself, this fact suggests that the pullulanase-type DBE is not the primary determinant of the PG-accumulating phenotype in maize endosperm. However, when combined with a specific allele of $s u 1$, sul-starchy (sul-st), the zpul null mutation causes a fivefold increase in the amount of PG. Thus there appears to be some functional overlap between the two DBE isoforms. ${ }^{6)}$

In this study, genetic methods were employed to eliminate pullulanase-type DBE activity in a series of maize lines containing various mutations of the sul gene, each with distinct effects on the isoamylase-type DBE and the PG accumulation phenotype. The data confirmed that pullulanase-type DBE can affect starch biosynthesis, because in the presence of several sul alleles that reduce but do not completely eliminate isoamylase-type DBE activity, the pullulanase-type DBE determined the amount of PG versus granular starch produced. Of further interest was to test the effects of combining null mutations of both zpul and sul in single plants, thus eliminating all detectable DBE proteins and activity in developing endosperm. This experiment revealed that the ability of pullulanase-type DBE to influence starch versus PG content requires the presence of a critical threshold level of isoamylase-type DBE activity or protein, indicative of a specific function for the latter isoform in starch biosynthesis. Furthermore, granular starch accumulated in the double mutants to the same extent observed in the null $s u 1^{-}$ single mutant, thus ruling out the possibility that residual granule formation in isoamylase-type DBE mutants results from partial substitution of an isoamylase-type DBE function by the pullulanase-type DBE activity.

\section{MATERIALS AND METHODS}

Plant material. All maize plants (Zea mays L.) used in this study were in the standard inbred W64A genetic background. The null mutations zpul-204 and sul-R4582::Mul (su1-4582) were described previously. ${ }^{6.11,22)}$ Seeds containing the allele sul-R5051::Mul (su1-5051) were provided by Dr. Donald S. Robertson (Iowa State University, Ames, IA), and su1-Brawn2 (su1-Bn2 $)^{23)}$ seeds were obtained from the Maize Genetics Cooperation Stock Center (Urbana, IL). All plants used in the study were grown under field conditions in Ames, IA.

Carbohydrate extraction and quantification. Mature, dried kernels were imbibed in $0.3 \%(\mathrm{w} / \mathrm{v})$ sodium metabisulfite, $1 \%(\mathrm{v} / \mathrm{v})$ lactic acid overnight at $50^{\circ} \mathrm{C}$. The pericarp and embryo were removed by manual dissection. Endosperm tissue from individual kernels was homogenized in a microfuge tube using a pestle and suspended in 0.5 $\mathrm{mL} \mathrm{H}_{2} \mathrm{O}$. The homogenate was centrifuged at $3000 \mathrm{~g}$ for $5 \mathrm{~min}$ at room temperature, and the pelleted material was resuspended with $0.5 \mathrm{~mL} \mathrm{H}_{2} \mathrm{O}$ and centrifuged two additional times. The supernatants from each centrifugation were pooled together. Endosperm starch and WSP in the pellet and supernatant fractions, respectively, were measured as described previously." Results are expressed as percent starch or WSP of total $\alpha$-glucan polymer per endosperm.

Kernel genotype determination. DNA was isolated from embryos of individual kernels following standard procedures. ${ }^{24)}$ The genotype was determined using polymerase chain reaction (PCR) amplification with primers specific for either the wild-type Zpul allele or the mutant zpu1-204 allele as described. ${ }^{6}$ )

\section{RESULTS AND DISCUSSION}

\section{Generation of double mutant kernels.}

To analyze the functional interaction between the maize pullulanase- and isoamylase-type DBEs, seeds carrying mutations affecting both enzyme isoforms were generated (Fig. 1). Singly homozygous maize plants bearing the pullulanase-type DBE mutation zpul-204 were crossed to plants homozygous for the isoamylase-type DBE mutant alleles su1-5051, su1-Bn2, or su1-4582. The progeny

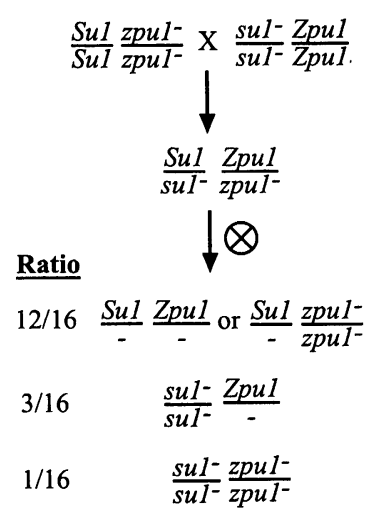

Fig. 1. Crossing scheme used to generate $\mathrm{sul}^{-} \mathrm{zpul}^{-}$double mutants.

Singly homozygous plants were.crossed and the resulting compound heterozygote was self-pollinated to give the $F_{2}$ ear. These ears segregate for the doubly homozygous mutant kernels in a ratio of approximately one-sixteenth. Dashes ( - ) indicate that either the nonmutant allele or the mutant allele can be present in kernels of the particular phenotypic class. 
plants from this cross were heterozygous at both the zpul and sul loci, so that their self-pollination generated ears on which kernels segregated independently for both mutations. One-fourth of the kernels are expected to be homozygous for the recessive $s u 1^{-}$mutant allele and thus to display a discernable sugary phenotype, i.e., shrunken kernels with a glassy appearance. One-fourth of the kernels also are expected to be homozygous for zpul-204, however, this defect does not condition a noticeable kernel phenotype. $\left.{ }^{6}\right)$ As expected, in all instances three-fourths of the kernels on the ear displayed a wild type appearance, and one-fourth displayed the sugary phenotype to varying degrees (Fig. 2).

The allele sul-5051 conditions a moderate phenotype in which kernels are slightly collapsed at the crown, and

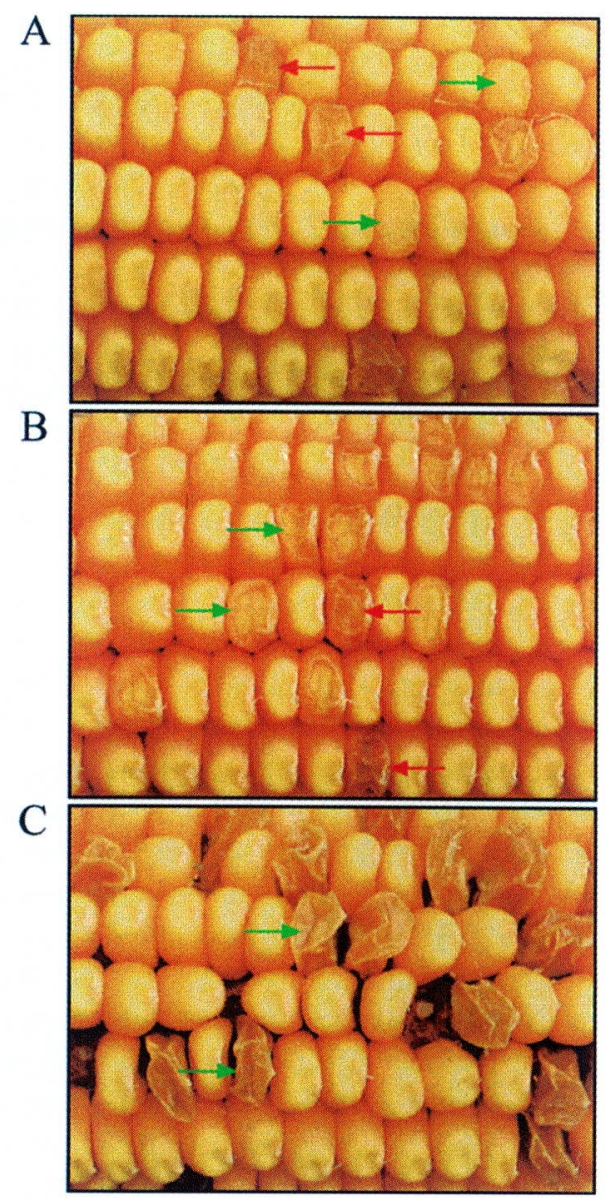

Fig. 2. Kernel phenotypes of $s u 1^{-} z p u 1^{-}$double mutants.

A) A portion of the ear resulting from the self pollination of a Sul/su1-5051; Zpu1/zpu1-204 compound heterozygote is shown. Approximately three-sixteenths of the kernels possess the moderate kernel phenotype typical of the sul-5051 homozygous parent (green arrows). A more severe sugary kernel phenotype appears in approximately one-sixteenth of the progeny (red arrows). B) A portion of the ear resulting from the self-pollination of a $S u 1 / s u 1-B n 2$; Zpul/zpul-204 compound heterozygote. The moderate kernel phenotype typical of the sul-Bn2 parent appears in approximately three-sixteenths of the progeny (green arrows) and a more severe phenotype occurs in about one-sixteenth of the progeny (red arrows). C) A portion of the ear resulting from the self-pollination of a Su1/su1-4582; Zpul/zpu1-204 compound heterozygote. Approximately one-fourth of the kernels possess the extremely shrunken and glassy kernel phenotype of the sul-4582 parent (green arrows). No obvious difference can be detected between the kernels that are also homozygous for zpul-204 and those that are not. this was observed in approximately three-sixteenths of the kernels on the ear (Fig. 2A, green arrows). One-sixteenth of the kernels displayed a more severe shrunken and glassy phenotype not typical of sul-505l mutants (Fig. 2A, red arrows), and these were presumed to be sul5051, zрu1-204 double homozygotes. The genotype at the zpul locus was determined by PCR amplification of genomic DNA $^{6}$ from embryos of five individual kernels with the moderate phenotype and five others with the more severe phenotype. In all instances the severe kernels were confirmed to be homozygous for zpul-204, whereas all of the less severely affected kernels contained at least one wild type Zpul allele (data not shown). The endosperm tissue from the same kernels that were used for genotype determination was saved for determination of granular and soluble glucan content. Thus this population of kernels allowed comparison of congenic endosperm tissue that differed only by the presence or absence of a functional Zpul allele.

Similar results were obtained for the kernel population segregating for $s u 1-B n 2$ and $z p u 1-204$, again with threesixteenths of the progeny displaying a relatively weak sugary phenotype (Fig. 2B, green arrows) and onesixteenth with an enhanced phenotype (Fig. 2B, red arrows). In this cross the enhanced phenotype was characterized by a more collapsed kernel crown and by a slightly darker color than the typical moderate phenotype conditioned by sul-Bn2. Genotype determination by PCR again confirmed that the more severely affected kernels were homozygous for zpu1-204 and that the less severely affected kernels all contained at least one wild type Zpul allele (data not shown). From the cross involving the null allele sul-4582 there was no visual distinction possible among the sugary kernels, all of which were severely affected to the same extent (Fig. 2C). Homozygous zpul204 kernels were distinguished by PCR analysis from sibling seeds that contained at least one copy of the functional Zpul allele (data not shown).

\section{Soluble and granular carbohydrate distribution in sin- gle kernels.}

As described above, the genotypes of mature, dried kernels were determined from analysis of the dissected embryos, and endosperm from the same individual kernels was used to determine the percentage of glucan polymers in the soluble and granular phases. In the cross involving su1-5051, the segregant kernels with wild type appearance (presumed to contain at least one wild type Sul allele) contained less than $1 \%$ of their total $\alpha$-glucan polymer in the form of WSP (Table 1). In previous analyses the WSP from wild type kernels was shown to be small maltooligosaccharides as opposed to $\mathrm{PG}^{22)}$ The $s u l-5051$ single mutant kernels (sul-5051/sul-5051; Zpul/Zpul or sul5051/su1-5051; Zpul/zpul-204) possessed higher levels of WSP than the wild type kernels, amounting to $13.4 \%$ of the total $\alpha$-glucan polymer, and accordingly exhibited a statistically significant reduction in the amount of starch present (Table 1). The double mutant kernels (sul-5051) su1-5051; zpul-204/zpu1-204) possessed markedly more WSP than the sul-5051 single mutants, approximately $50 \%$ of the total $\alpha$-glucan polymer (Table 1). These data 
Table 1. Carbohydrate content of individual kernels. ${ }^{*}$

\begin{tabular}{|c|c|c|c|c|}
\hline Kernel genotype & $\begin{array}{c}\text { No. of } \\
\text { kernels } \\
\text { analyzed }\end{array}$ & $\begin{array}{c}\% \\
\text { Starch }\end{array}$ & $\begin{array}{c}\% \\
\text { WSP }\end{array}$ & $\begin{array}{l} \pm \\
\mathrm{SD}\end{array}$ \\
\hline \multicolumn{5}{|l|}{ Segregants of $s u 1-5051 \times z p u l-204:$} \\
\hline Sul/-; Zpul/- or Sul/-; zpu1-/zpu1- & 5 & 99.4 & 0.6 & 0.3 \\
\hline sul-/sul-; Zpul/- & 5 & 86.6 & 13.4 & 2.0 \\
\hline suI $I^{-/ s u I^{-} ; z p u I^{-} / z p u I^{-}}$ & 5 & 50.3 & 49.7 & 5.8 \\
\hline \multicolumn{5}{|l|}{ Segregants of $s u I-B n 2 \times z p u l-204:$} \\
\hline Sul/-; Zpul/- or Sul/-; zpul-/zpul- & 5 & 98.8 & 1.2 & 0.3 \\
\hline sul-/sul-; Zpul/- & 7 & 74.9 & 25.1 & 6.4 \\
\hline$s u I^{-} / s u l^{-} ;$zpul-/zpul- & 3 & 53.1 & 46.9 & 1.3 \\
\hline \multicolumn{5}{|l|}{ Segregants of sul-4582 $\times z p u 1-204$ : } \\
\hline sul-/sul-; Zpul/- & 15 & 37.8 & 62.2 & 8.3 \\
\hline $\mathrm{sul}^{-} / \mathrm{sul} \mathrm{I}^{-} ; \mathrm{zpul} \mathrm{I}^{-} / \mathrm{zpul}^{-}$ & 5 & 36.3 & 63.7 & 6.0 \\
\hline
\end{tabular}

*Values for percent starch and percent WSP are normalized to the total glucan polymer content in each individual kernel that was analyzed.

suggest that in the sul-5051 mutant, the pullulanase-type DBE prevents accumulation of large amounts of PG and thereby partially substitutes for the reduction in isoamylase-type DBE. An increase in PG content of about this magnitude was also observed previously in the sul-st; zpul-204 double mutant compared to the sul-st single mutant. ${ }^{6)}$

Similar results were obtained from the crosses between zpul-204 plants and those bearing a slightly more severe $\mathrm{sul}^{-}$allele, su1-Bn2. Wild type kernels from this cross were again found to contain very low levels of WSP, approximately $1 \%$ of the total $\alpha$-glucan polymer (Table 1 ). The single mutant kernels (sul-Bn2/su1-Bn2; Zpu1/Zpul or sul-Bn2/sul-Bn2; Zpu1/zpu1-204) were found to contain $25.1 \%$ WSP, whereas the double mutant kernels (sulBn2/sul-Bn2; zpul-204/zpu1-204) contain approximately twice that amount. Although the two-fold increase is modest compared to the results of coupling zpul-204 with sul5051 (Table 1) or sul-st, ${ }^{6)}$ the difference clearly is statistically significant $(p<0.05)$.

To specifically examine the effect on starch metabolism of complete loss of both isoamylase- and pullulanase-type DBE, crosses were made to generate double null mutant seeds. The sul-4582 mutation contains a $M u 1$ transposable element in the first exon of the gene. ${ }^{11)}$ As expected, mRNA transcript is undetectable in seeds homozygous for this allele, ${ }^{22}$ nor is any isoamylase-type DBE activity observed in developing mutant endosperm (J. Dinges and C. Colleoni, unpublished results). On segregating ears, there was no apparent phenotypic difference between kernels homozygous for only sul-4582 and those homozygous for mutations at both the sul and zpul loci (Fig. 2C). Singlekernel carbohydrate analysis and genotyping of 20 sugary kernels from a segregating ear revealed those seeds which were, in fact, double mutants (Table 1). The single mutant sul-4582 kernels contained $62.2 \%$ WSP and the double mutant su1-4582; zpul-204 kernels contained 63.7\% WSP (Table 1), a difference that is not statistically significant $(p>0.05)$. Thus, in the case of the sul-4582 mutation, the presence or absence of pullulanase-type DBE activity does not affect the level of PG versus granular starch produced.

Interaction between isoamylase- and pullulanase-type DBEs.

This study made use of three allelic mutations in the sul locus, which differ in the severity of the phenotype that results as it is measured both by the visible morphology and the ratio of WSP to granular starch. The most severe mutation is the null allele sul-4582, which completely eliminates the mRNA, protein, and enzymatic activity that correspond to this particular isoamylase-type DBE. The molecular defect in sul-505I is caused by a Mul transposable element inserted into the $5^{\prime}$ untranslated region, at nucleotide position 1964 of the published genomic sequence (Genbank accession no. AF030882) (J. Dinges, unpublished data). Genes with such a structure are termed $M u$-suppressible, ${ }^{25)}$ because transcription is not entirely eliminated. Rather, a promoter within the $M u$ element allows for transcription at a level that is reduced compared to wild-type (data not shown). Thus sul-505I is likely to result in diminished production of the SU1 protein relative to wild type, but that protein should have the normal amino acid sequence. The molecular defect of $s u 1-B n 2$ is a single nucleotide change resulting in substitution of the asparagine at position 627 by a lysine residue (J. Dinges, unpublished results). In this instance, therefore, normal gene expression is expected, although the product will have an altered primary sequence.

Elimination of pullulanase-type DBE activity had an effect on starch content in the presence of the two weaker $s u 1^{-}$alleles, su1-5051 and su1-Bn2, but not in su1-4582 kernels completely lacking sul function. Based on these results, we conclude that some degree of isoamylase-type DBE function is required in order for the pullulanase-type DBE to be able to affect starch biosynthesis. Above a predicted threshold level the glucan biosynthesis system might proceed to the point where further $\alpha(1 \rightarrow 6)$ glucan hydrolase activity from the pullulanase-type DBE could cause a higher degree of partitioning of polymer into the granular phase, whereas below this level either pullulanase-type DBE activity is not possible, or is not productive in terms of granule formation. A possible explanation of this finding is that there is a critical level of isoamylase-type DBE activity that is needed in order to form a substrate on which the pullulanase-type DBE could act. Alternatively, the isoamylase-type DBE protein might have a direct or indirect effect on the enzymatic properties of the pullulanase-type DBE. The alleles that produce either reduced levels of the sul gene product, or a protein with an altered primary sequence, would be able to provide the requisite isoamylase-type DBE function, whereas the null allele would not.

The data reported here confirm the previous conclusion that the isoamylase-type DBE and the pullulanase-type $\mathrm{DBE}$ are capable of working synergistically in normal kernels to prevent larger amounts of PG from accumulating. It is not obvious, therefore, why the complete or nearly complete loss of DBE activity in the sul-4582; zpul-204 double homozygotes does not result in the complete replacement of granular starch by PG. Three reasons might be offered to explain starch formation in the double mutant kernels. First, starch synthesis may be a completely separate process from PG accumulation, as suggested in the WSP clearing model proposed by Zeeman and colleagues. ${ }^{13)}$ DBE activity may not be needed for the formation of granular starch, but instead it may be re- 
quired for preventing the diversion of substrate and enzyme to synthesis in the soluble phase. The approximately $40 \%$ residual starch present in the double mutant may be the equilibrium point in the competiton for glucan synthesis in the soluble and granular phases. A second explanation for the residual starch is that some degree of isoamylase-type DBE function is being provided by one or both of the two additional genes present in the maize genome that code for members of this enzyme class. Although their activities have not been observed on native PAGE/activity gels, the possibility exists that these enzymes are contributing to starch metabolism in the maize endosperm. Such activity might be sufficient to allow for low level granular starch formation, but not provide whatever minimal function is needed to enable the pullulanasetype DBE to contribute further to the formation of granular starch. Finally, the remaining starch in the sul-4582; zpu1-204 endosperm may be created due to random crystallization events that occur at the surface of the starch granule. Myers et al ${ }^{17)}$ speculated that crystallization would be an entropically-driven process, which would be facilitated by removal of extraneous branches by DBEs. The model predicts that some crystallization could occur in the absence of DBE activity.

Although these data show that both DBE isoform classes influence the degree of phytoglycogen accumulation, they do not distinguish the exact nature of the overall function of DBEs in starch synthesis, nor do they imply that both DBE isoforms necessarily must have the same function. An overlapping function in a single step in starch biosynthesis is consistent with the data. Separate functions, however, also are consistent with the observations. For example, a role for the isoamylase-type DBE in glucan trimming does not preclude the pullulanase-type DBE from acting in WSP clearing, or visa versa. Thus the possibility exists that the two DBE isoforms are involved in two distinct processes, both of which contribute to the partitioning of glucans between the soluble- and the granular phases.

\section{REFERENCES}

1 ) E.Y.C. Lee and W.J. Whelan: Glycogen and starch debranching enzymes. in The Enzymes, P. Boyer, ed., Academic Press, New York, pp. 191-234 (1971).

2 ) D.C. Doehlert and C.A. Knutson: Two classes of starch debranching enzymes from developing maize kernels. J. Plant Physiol., 138, 566-572 (1991).

3 ) C. Wu, C. Colleoni, A.M. Myers and M.G. James: Enzymatic properties of ZPU1, the maize pullulanase-type starch debranching enzyme. Arch. Biochem. Biophys., 406, 21-32 (2002).

4 ) S. Tabata, T. Kaneko, Y. Nakamura et al.: Sequence and analysis of chromosome 5 of the plant Arabidopsis thaliana. Nature, 408, 823-826 (2000).

5 ) J. Yu, S. Hu, J. Wang et al.: A draft sequence of the rice genome (Oryza sativa L. ssp. indica). Science, 296, 79-92 (2002).

6 ) J.R. Dinges, C. Colleoni, M.G. James and A.M. Myers : Mutational analysis of the pullulanase-type debranching enzyme of maize indicates multiple functions in starch metabolism. Plant Cell, 15, 666-680 (2003).

7 ) A. Renz, S. Schikora, R. Schmid, J. Kossmann and E. Beck:
cDNA sequence and heterologous expression of monomeric spinach pullulanase: Multiple isomeric forms arise from the same polypeptide. Biochem. J., 331, 937-945 (1998).

8 ) M.K. Beatty, A. Rahman, H. Cao, W. Woodman, M. Lee, A. M. Myers and M.G. James: Purification and molecular genetic characterization of ZPU 1, a pullulanase-type starchdebranching enzyme from maize. Plant Physiol., 119, 255-266 (1999).

9 ) M. Kristensen, F. Lok, V. Planchot, I. Svendsen, R. Leah and B. Svensson: Isolation and characterization of the gene encoding the starch debranching enzyme limit dextrinase from germinating barley. Biochim. Biophys. Acta, 1431, 538-546 (1999).

10) S.A. Goff, D. Ricke, T.H. Lan et al.: A draft sequence of the rice genome (Oryza sativa L. ssp. japonica). Science, 296, 92100 (2002).

11) M.G. James, D.S. Robertson and A.M. Myers: Characterization of the maize gene sugaryl, a determinant of starch composition in kernels. Plant Cell , 7, 417-429 (1995).

12) N. Fujita, A. Kubo, P.B. Francisco, Jr., M. Nakakita, K. Harada, N. Minaka and Y. Nakamura: Purification, characterization, and cDNA structure of isoamylase from developing endosperm of rice. Planta, 208, 283-293 (1999).

13) S.C. Zeeman, T. Umemoto, W.L. Lue, P. Au-Yeung, C. Martin, A.M. Smith and J. Chen: A mutant of Arabidopsis lacking a chloroplastic isoamylase accumulates both starch and phytoglycogen. Plant Cell, 10, 1699-1712 (1998).

14) R.A. Burton, H. Jenner and L. Carrangis et al.: Starch granule initiation and growth are altered in barley mutants that lack isoamylase activity. Plant J., 31, 97-112 (2002).

15) G. Mouille, M.-L. Maddelein, N. Libessart, P. Talaga, A. Decq, B. Belrue and S. Ball: Preamylopectin processing: a mandatory step for starch biosynthesis in plants. Plant Cell, $\mathbf{8}$, 1353-1366 (1996).

16) S. Ball, H.P. Guan, M. James, A. Myers, P. Keeling, G. Mouille, A. Buleon, P. Colonna and J. Preiss: From glycogen to amylopectin: A model for the biogenesis of the plant starch granule. Cell, 86, 349-352 (1996).

17) A.M. Myers, M.K. Morell, M.G. James and S.G. Ball: Recent progress toward understanding the biosynthesis of the amylopectin crystal. Plant Physiol., 122, 989-998 (2000).

18) A.M. Smith: The biosynthesis of starch granules. Biomacromology, 2, 335-341 (2001).

19) D. Pan and O.E. Nelson, Jr.: A debranching enzyme deficiency in endosperms of the sugaryl mutants of maize. Plant Physiol., 74, 324-328 (1984).

20) Y. Nakamura, A. Kubo, T. Shimamune, T. Matsuda, K. Harada and H. Satoh: Correlation between activities of starch debranching enyzme and alpha-polyglucan structure in endosperms of sugary-1 mutants of rice. Plant J., 12, 143-153 (1997).

21) A. Kubo, N. Fujita, K. Harada, T. Matsuda, H. Satoh and Y. Nakamura: The starch-debranching enzymes isoamylase and pullulanase are both involved in amylopectin biosynthesis in rice endosperm. Plant Physiol., 121, 399-410 (1999).

22) J.R. Dinges, C. Colleoni, A.M. Myers and M.G. James: Molecular structure of three mutations at the maize sugaryl locus and their allele-specific phenotypic effects. Plant Physiol., 125, 1406-1418 (2001).

23) R.A. Brink: Maize endosperm mutants affecting soluble carbohydrate content as potential addititives in preparing silage from high protein forages. Maydica, 29, 265-286 (1984).

24) M.A. Saghai-Maroof, K.M. Soliman, R.A. Jorgensen and R.W. Allard: Ribosomal DNA spacer-length polymorphisms in barley. Proc. Natl. Acad. Sci. USA, 81, 8014-8018 (1984).

25) A. Barkan and R.A. Martienssen: Inactivation of maize transposon $\mathrm{Mu}$ suppresses a mutant phenotype by activating an outward-reading promoter near the end of Mu1. Proc. Natl. Acad. Sci. USA, 88, 3502-3506 (1991). 